\title{
PRESSURE LOSS ANALYSIS IN THE STEAM TURBINE CONTROL VALVE
}

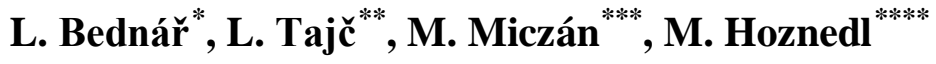

\begin{abstract}
Pressure loss analysis is carried out in the sub parts of the control valve. Attention is devoted to the pressure loss on protective strainer, to the pressure loss in valve chamber and in the diffuser. The influence of the flow vortex under the cone on the loss in the diffuser is analysed. The flow in the valve is considered for specific operational characteristics of the turbine.
\end{abstract}

Keywords: Control valve, pressure loss, steam turbine.

\section{Introduction}

Control valves must ensure the mass flow of the steam required to drive the turbine at the required output level. It can be reached by a change of flow area in the valve and by adjustment of pressure ratio. With reduced turbine output, pressure reduction is necessary. Certain pressure and energetic loss occurs in the valve. With the full output, it is desirable for the loss to be the lowest. The pressure loss in the valve consists of a number of partial losses. The aim of the work is to map their path in the whole course of turbine operation and to make recommendations for minimizing losses with maximum turbine output and a totally open valve.

\section{Loss pressure in the valve}

The characteristic design of control valve is shown in Fig. 4. The steam enters the valve chamber through the protective strainer. The valve chamber is connected with the outlet diffuser via a saddle. Mass flow $G$ is controlled by the change of the flow area, which is made possible by the lift of cone $h$. For each valve type it is possible to define general characteristics of the flow. An example of such characteristics can be found in Fig. 1. It shows the dependence of proportional flow $q=G / G *$ on the pressure ratio $\varepsilon_{2}=p_{2} / p_{0 c}$ at the given average stroke $\overline{\mathrm{h}}=\mathrm{h} / \mathrm{D}_{1}$. The real mass flow $G$ is related to the critical $G_{*}$, which is considered for steam inlet parameters and for the diffuser throat area. When connecting the control valve with the turbine, states are applied in general flow characteristics that are valid for operational characteristics. With the lowered turbine output the pressure in the valve is throttled to the lowest value. The pressure loss is necessary in this process. As for the function, the focus is mainly on operational reliability. With the valve fully open the pressure loss is required to be the lowest. The graph in Fig. 2 shows how the proportional pressure loss $\Delta \mathrm{p}_{\mathrm{z}} / \mathrm{p}_{0 \mathrm{c}}$ changes in dependence on the proportional cone stroke. The pressure throttling process in the valve is shown in Fig. 3. Here the pressure loss from the step change of cross section at the valve inlet is applied as well as the pressure loss on the protective strainer and also the loss caused by friction and the geometrical arrangement of the valve chamber. Steam expansion continues up to the diffuser throat to the pressure level $\mathrm{p}_{1}$. In this section mainly the pressure loss caused by friction on the saddle wall is applied. There is also the influence of cross section step

\footnotetext{
Ing. Lukáš Bednář: Doosan Škoda Power s. r. o., Tylova 1/57, 30128 Plzeň; CZ, lukas.bednar@doosan.com

** Ing. Ladislav Tajč, CSc.: Doosan Škoda Power s. r. o., Tylova 1/57, 30128 Plzeň; CZ, ladislav.tajc@doosan.com

**** Ing. Martin Miczán, Ph.D.: Doosan Škoda Power s. r. o., Tylova 1/57, 30128 Plzeň; CZ, martin.miczan@doosan.com

**** Doc. Ing. Michal Hoznedl, Ph.D.: Doosan Škoda Power s.r.o., Tylova 1/57, 30128 Plzeň; CZ, michal.hoznedl@doosan.com
} 
change in the passage from the valve saddle to the diffuser here. In the diffuser the loss by friction is applied as well as by the cross section enlargement, and there is also the influence of flow vortex under the cone. At the valve outlet even the pressure loss can be applied caused by the transition to the pipe of different diameter from the one at the diffuser outlet.

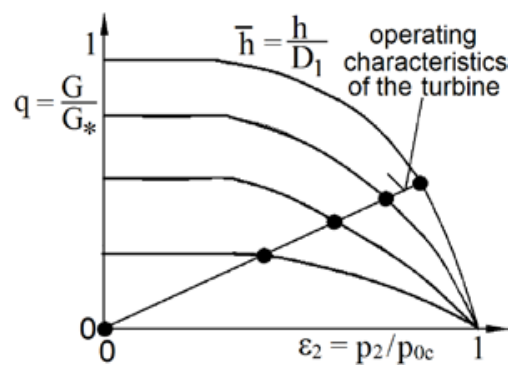

Fig. 1: Valve flow characteristics

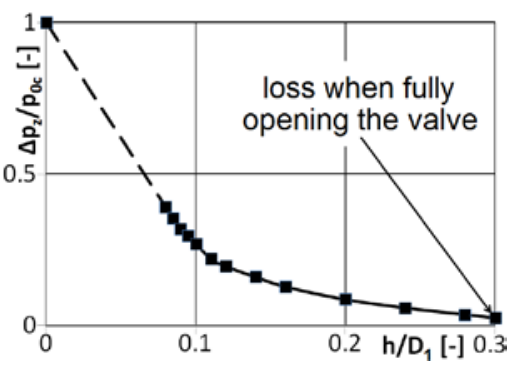

Fig. 2: Proportional pressure loss on the valve

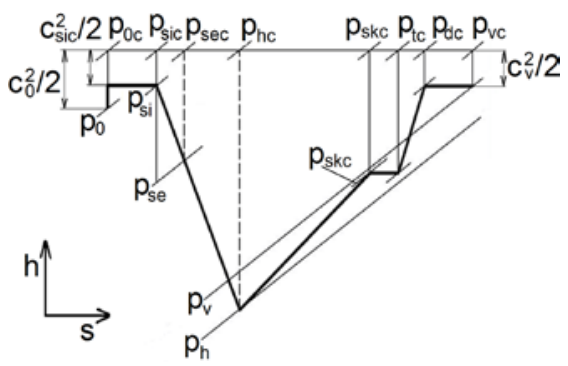

Fig. 3: Pressure throttling process in the valve

\section{Analysis of pressure losses at the valve}

\subsection{Pressure loss at the valve chamber inlet}

The inner flow area of the valve chamber is usually larger than the inlet pipe area, which leads to the occurrence of pressure loss caused by a sudden enlargement of the cross section. The flow characteristics on the valve chamber inlet are shown in Fig. 4 . The pressure loss is defined as a quotient of inlet dynamic pressure

$$
\Delta p_{s k}=\zeta_{s k} \frac{1}{2} \rho_{0} c_{0}^{2} \text {, where } \zeta_{s k}=(0,8 \div 1)\left(1-\frac{1}{n_{1}}\right)^{2}
$$

Here $n_{1}$ means the stage of area enlargement. The greater the steam velocity in the pipeline, the larger the pressure loss. In Fig. 5 proportional mass flow through the valve is processed according to experiments (Zarjankin, 2005) for two inlet steam velocities. Adjusting the inlet pipe decreased the inlet velocity from $80 \mathrm{~m} / \mathrm{s}$ (curve 1) to $40 \mathrm{~m} / \mathrm{s}$ (curve 2). The most significant changes are evident in the fully open valve with $\overline{\mathrm{h}}=0.34$, where with $\mathrm{q}=0.4$ the pressure loss dropped by $0.8 \%$ of the inlet pressure.

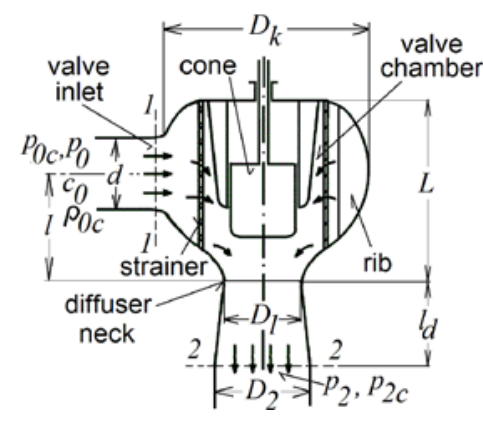

Fig. 4: Control valve design, diagram of the basic geometric parameters of the valve and flow in the valve chamber

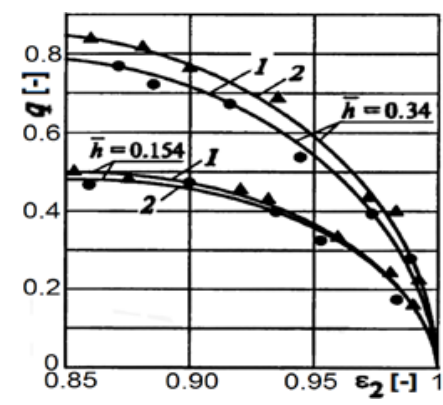

Fig. 5: Influence of inlet velocity on losses

\subsection{Loss pressure in the protective strainer}

Based on experiments (Miczan, 2016) the dependence of the strainer loss coefficient $\zeta_{s i}$ on Reynolds number is processed for a standard strainer design. It is found in Fig. 6. The pressure loss in the strainer is defined as

$$
\Delta p_{s i}=k_{s i} \zeta_{s i} \frac{1}{2} \rho c_{s i}^{2}
$$

Here $\mathrm{k}_{\mathrm{si}}$ is the correction on the influence of $3 \mathrm{D}$ flow inside the valve chamber. It is evident that the real pressure loss in the strainer can be as much as 3times bigger than its theoretical value for 2D flow. The share of the pressure loss that occurred in the strainer on the total pressure loss is shown in Fig. 7. With a fully open valve the loss in the strainer may represent up to $30 \%$ of the total loss. 


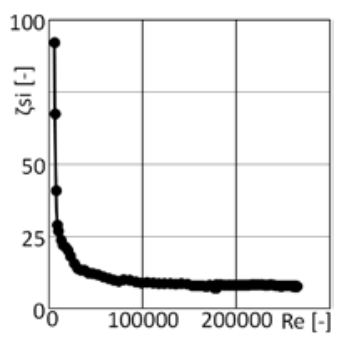

Fig. 6: Loss coefficient of the strainer

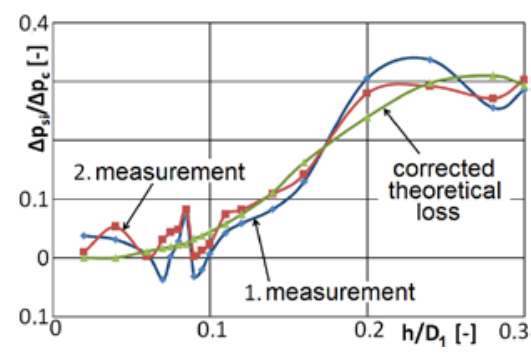

Fig. 7: Share of strainer pressure loss in the total pressure loss

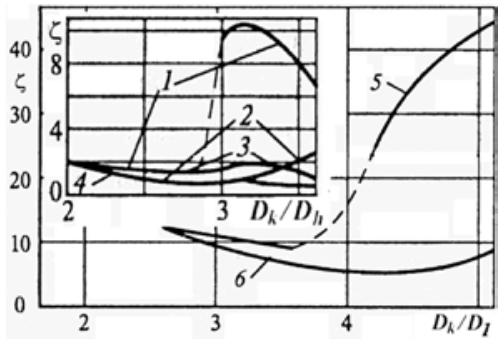

Fig. 8: Influence of valve chamber dimension on the loss coefficient value

\subsection{Pressure loss in the valve chamber}

The pressure loss in the valve chamber can be influenced by its dimensions, the shaping of the flow part. The designation of the dimensions of the individual parts of the valve is in Fig. 4. In Fig. 8 there is the course valve chamber loss coefficient $\zeta_{\mathrm{vk}}$ for various configurations of the chamber depending on the ratio $\mathrm{D}_{\mathrm{k}} / \mathrm{D}_{1}$. Here the pressure loss is calculated to the dynamic pressure in the inlet pipeline. The optimal design of the valve chamber requires $\mathrm{D}_{\mathrm{k}} / \mathrm{D}_{1} \approx 4$. It is necessary to use a dividing rib (see Fig. 4) on the side opposite the inlet. The dividing rib prevents the occurrence of circulating flow, when the loss coefficient $\zeta_{\mathrm{vk}}$ increases significantly. This corresponds with states 1 and 5 in Fig. 8.

From the measurement and computational studies (Turecky, 2015) it is known that steam expansion in the valve goes up to the diffuser throat, where pressure $p_{1}$ is measured. The pressure loss in the valve chamber is thus considered for the drop from the total pressure in the strainer $\mathrm{p}_{\text {sic }}$ to pressure $\mathrm{p}_{1}$ as shown in Fig. 3.

$$
\Delta p_{v k}=\zeta_{v k}\left(p_{s i c}-p_{1}\right)
$$

The loss in the valve chamber in dependence on the cone stroke for operational characteristics of the turbine is in Fig. 9. The pressure loss in the chamber increases with gradual opening of the valve and when the valve saddle area equals the diffuser throat area it reaches the maximum value, which is about $11 \%$ of the total loss.

\subsection{Pressure loss in the diffuser}

In references, e.g. (Povch, 1974) it is possible to find the basis for the calculation of pressure loss in diffusers with various angles and degrees of enlargement. Ideal velocity ratios are considered in the diffuser inlet. However, flow vortex occurs under the cone in the valves, and in the diffusers a backflow zone appears. For the calculation of the pressure loss in the diffuser it is necessary to adjust the velocity field, to divide it into the section uninfluenced by vortex and the section with vortex and with the influence of the flow cross section step change.

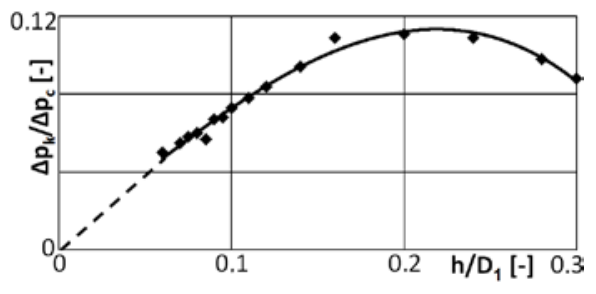

Fig. 9: Proportional pressure loss in the valve chamber
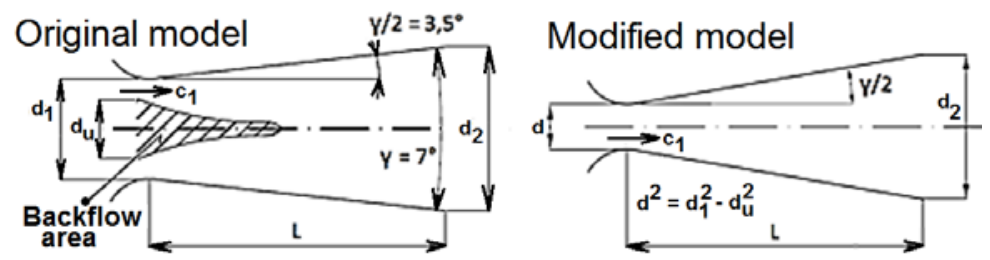

Fig. 10: Adjustment of the diffuser computational model

If the vortex section is displaced as shown in Fig. 10, the recommended relations can be used for pressure loss calculation. The diffuser loss is considered as a loss by a step change of the cross section with an adjustment using the mitigation coefficient $\varphi_{\mathrm{d}}$.

$$
\Delta p_{d}=\varphi_{\mathrm{d}}\left(1-\frac{1}{n_{2}}\right)^{2}\left(p_{1 c}-p_{1}\right)
$$


where $n_{2}=\left(d_{2} / d\right)^{2}$. The mitigation coefficient has two parts, the friction component $\varphi_{t}$ and the extension component $\varphi_{\mathrm{r}}\left(\varphi_{\mathrm{d}}=\varphi_{\mathrm{t}}+\varphi_{\mathrm{r}}\right)$.

$$
\varphi_{\mathrm{t}}=\frac{\lambda}{8 \sin \frac{\gamma}{2}} \cdot \frac{n_{2}+1}{n_{2}-1} \text { a } \varphi_{\mathrm{r}}=3,2\left(\operatorname{tg} \frac{\gamma}{2}\right)^{1,25}
$$

The course of individual pressure losses is processed in the graph in Fig. 11. The loss caused by friction on the diffuser wall is very low. The pressure loss caused by diffuser enlargement is of a greater importance. A share of loss is caused by the cross section step change. The missing share in the total pressure loss goes to the cross section step change between the diffuser inlet and outlet and also to the influence of flow vortex under the cone.

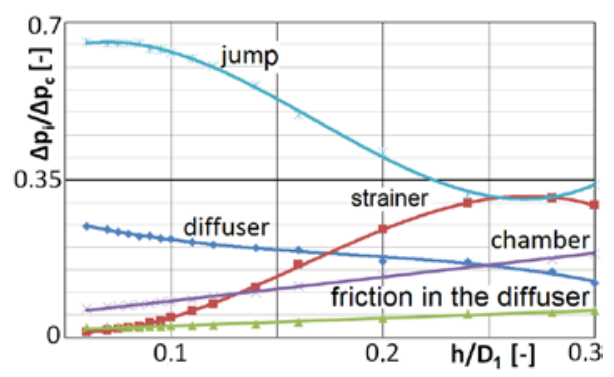

Fig. 11: Share of partial pressure losses in the valve.

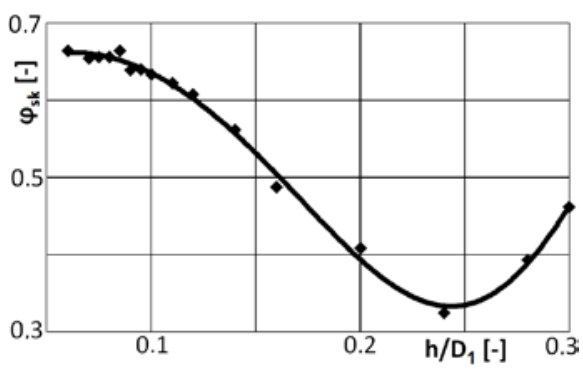

Fig. 12: Mitigation coefficient of pressure loss from the cross section step change.

\subsection{Pressure loss from the cross section step change.}

Here enlargement is considered of the diffuser saddle area $S_{\text {se }}$ to equal the diffuser outlet area. Mitigation coefficient $\varphi_{\text {sk }}$ applies here. The pressure loss is given by the relation

$$
\Delta p_{s k}=\varphi_{\mathrm{sk}}\left(1-\frac{1}{n_{v}}\right)^{2}\left(p_{h v}-p_{h 1}\right) \text {, where } n_{v}=\frac{\pi D_{2}^{2}}{4 S_{s e}}
$$

Pressure loss $\Delta \mathrm{p}_{\mathrm{sk}}$ constitutes the complementary pressure loss to $100 \%$ of the total loss after deduction of the already considered partial pressure losses. It is shown in Fig. 11. Using equation (6) the mitigation coefficient $\varphi_{\mathrm{sk}}$ is defined as shown in Fig. 12. In the initial phase of the cone stroke the pressure loss is caused mainly by the influence of the cross section step change between the saddle and the diffuser outlet and also by the influence of the diffuser enlargement. The loss is the lowest when the valve saddle area equals the diffuser throat area. The only influence applied here is the unbalanced velocity profile caused by the flow vortex under the cone. For the next cone stroke the resulting pressure loss due to cross section step change is invariable. However, as the saddle area for the next stroke further increases and $n_{v}$ drops, the mitigation coefficient $\varphi_{\text {sk }}$ goes up.

\section{Conclusions}

The total pressure loss in the control valve is constituted by a number of partial losses. At the turbine start with the minimal output the pressure loss in the valve is necessary and the focus is mainly on operational reliability. However, for the maximum turbine output the pressure loss in the valve is required to be minimal. Here the emphasis is on the optimal design of the valve. • The pressure loss in the valve can be influenced by the choice of valve dimensions and thus by the choice of velocity ratios in its flow part. • With a fully opened valve up to $30 \%$ of the total pressure loss falls on the protective strainer. $\bullet$ The same share of pressure loss is caused by unbalanced velocity profile in the diffuser inlet.

\section{References}

Zarjankin A. E. (2005) Regulirujushie i stoporno-regulirujushie klapany parovykh turbin, Publishing House MEI, Moscow.

Miczan M. (2016) The analysis of the force conditions and pressure losses in the unloaded control valve of the steam turbines, Dissertation, CTU Prague.

Turecky P. (2015) Rozbor tlakových ztrát v difuzoru regulačního ventilu, Thesis, CTU Prague.

Povch I. L. (1974) Aerodinamicheskijj eksperiment v mashinostroenii, Leningrad "mashinostroenie”, Leningradskoe otdelenie. 